\title{
AN INEQUALITY RELATED TO THE ISOPERIMETRIC INEQUALITY
}

\author{
L. H. LOOMIS AND H. WHITNEY
}

In this note we shall prove the following theorem.

TheOREM 1. Let $m$ be the measure of an open subset $O$ of Euclidean $n$-space, and let $m_{1}, \cdots, m_{n}$ be the $(n-1)$-dimensional measures of the projections of $O$ on the coordinate hyperplanes. Then

$$
m^{n-1} \leqq m_{1} m_{2} \cdots m_{n} .
$$

Note that for $n$-dimensional intervals with faces parallel to the coordinate hyperplanes, (1) holds with the equality sign.

With any reasonable definition of the $(n-1)$-dimensional measure $s$ of the boundary of $O, s \geqq 2 m_{i}$ for each $i$, so that (1) gives

$$
m^{n-1} \leqq s^{n} / 2^{n}
$$

this is the isoperimetric inequality, without the best constant. Since the proof of the isoperimetric inequality with the best constant is difficult, ${ }^{1}$ and since its applications do not necessarily require the best constant, our elementary proof of the theorem may be of some interest.

We first reduce the problem to a combinatorial one, in the following theorem.

THEOREM 2. Let $S$ be a set of cubes from a cubical subdivision of $n$-space; let $S_{i}$ be the set of $(n-1)$-cubes obtained by projecting the cubes of $S$ onto the ith coordinate hyperplane. Let $N$ and $N_{i}$ be the numbers of cubes in $S$ and $S_{i}$ respectively. Then

$$
N^{n-1} \leqq N_{1} N_{2} \cdots N_{n}
$$

Assuming Theorem 2, we prove Theorem 1 as follows. Given $\epsilon>0$, choose a cubical subdivision of $n$-space into cubes of side $\delta$, with $\delta$ so small that if $S$ is the set of cubes interior to $O$ forming the set $\bar{S}$, $\mu(O-\bar{S})<\epsilon(\mu=$ measure $)$. Then

$$
[\mu(\bar{S})]^{n-1}=N^{n-1} \delta^{n(n-1)} \leqq\left(N_{1} \delta^{n-1}\right) \cdots\left(N_{n} \delta^{n-1}\right) \leqq m_{1} \cdots m_{n},
$$

and since $\epsilon$ is arbitrary, (1) follows.

Received by the editors July 31, 1948.

${ }^{1}$ See E. Schmidt, Über das isoperimetrische Problem in Raum von $n$ Dimensionen, Math. Zeit. vol. 44 (1939) pp. 689-788. 
Proof of Theorem $2 .{ }^{2}$ If $n=2$, the theorem is clear; we shall use induction on $n$. Each cube of $S$ projects into an interval on the first coordinate axis; let $I_{1}, \cdots, I_{k}$ be the intervals thus obtained. Let $T_{i}$ be the set of cubes projecting onto $I_{i}$, and let $T_{i j}$ be the set of $(n-1)$ cubes obtained by projecting the cubes of $T_{i}$ into the $j$ th coordinate hyperplane $(j=2, \cdots, n)$. Let $a_{i}$ and $a_{i j}$ be the numbers of cubes in $T_{i}$ and $T_{i j}$ respectively. Clearly

$$
\begin{aligned}
\sum_{i=1}^{k} a_{i} & =N, \quad a_{i} \leqq N_{1}(i=1, \cdots, k), \\
\sum_{i=1}^{k} a_{i j} & =N_{j} \quad(j=2, \cdots, n) .
\end{aligned}
$$

Also, by induction,

$$
a_{i}^{n-2} \leqq a_{i 2} \cdots a_{i n} \quad(i=1, \cdots, k) .
$$

From (6) and the second part of (4) we obtain

$$
a_{i}^{n-1} \leqq N_{1} a_{i 2} \cdots a_{i n} \quad(i=1, \cdots, k) .
$$

Now using successively the first part of (4), the above inequality, Hölder's inequality, and (5), we see that

$$
\begin{aligned}
N & =\sum_{i=1}^{k} a_{i} \leqq \sum_{i=1}^{k} N_{1}^{1 /(n-1)} \prod_{j=2}^{n} a_{i j}^{1 /(n-1)} \\
& \leqq N_{1}^{1 /(n-1)} \prod_{j=2}^{k}\left(\sum_{i=1}^{m} a_{i j}\right)^{1 /(n-1)}=\prod_{j=1}^{n} N_{j}^{1 /(n-1)},
\end{aligned}
$$

as required.

\section{HARVARD UNIVERSITY}

${ }^{2}$ The authors are indebted to M. R. Demers for a simplification in the proof of this theorem. 